\title{
Editorial: Metabolism and Target Organ Damage
}

\author{
Amedeo Lonardo \\ Operating Unit of Metabolic Syndrome, Azienda Ospedaliero-Universitaria di Modena, Ospedale Civile di Baggiovara, Modena \\ 41126, Italy.
}

Correspondence to: Prof. Amedeo Lonardo, Department of Internal Medicine, Ospedale Civile di Baggiovara, 1135 Via Giardini, Modena 41126, Italy. E-mail: a.Ionardo@libero.it

How to cite this article: Lonardo A. Editorial: Metabolism and Target Organ Damage. Metab Target Organ Damage 2021;1:1. http://dx.doi.org/10.20517/mtod.2021.01

Received: 11 Mar 2021 Accepted: 11 Mar 2021 Available online: 15 Mar 2021

Academic Editor: Amedeo Lonardo Copy Editor: Xi-Jun Chen Production Editor: Xi-Jun Chen

Dear colleagues, ladies and gentlemen,

The concept inspiring the logo of our journal's cover [Figure 1] is "Metabolism" defined as the complex set of chemical reactions which are finalized to maintain the integrity of bodily tissues and to produce energy from food.

A metabolic disease will occur whenever abnormal chemical reactions will disrupt the process of physiological bio-transformations in our body. As a result, either some chemicals will accumulate or other ones that are necessary to stay healthy will become deficient.

Storage of excess energy in the form of inert fat in the adipose tissue leads to obesity. However, failure to accommodate all excess energy in the adipose tissue will lead to spillover of this fat in non-adipose tissues such as the liver, muscles and pancreas, thereby eventually leading to target organ damage. This pathogenic mechanism is defined as lipotoxicity ${ }^{[1]}$.

Although there are different groups of disorders that affect the metabolism of individual classes of nutrients (such as amino acids, carbohydrates, and lipids), in clinical practice the most commonly observed one among metabolic disorders is "Metabolic syndrome". This definition identifies the cluster of impaired glucose disposal, arterial hypertension and dyslipidemia that often result from abdominal obesity. Impaired intracellular transmission of insulin signaling, which characterizes insulin resistance, is the shared common denominator of many patients with either incomplete or full-blown metabolic syndrome $e^{[2]}$.

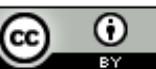

(C) The Author(s) 2021. Open Access This article is licensed under a Creative Commons Attribution 4.0 International License (https://creativecommons.org/licenses/by/4.0/), which permits unrestricted use, sharing, adaptation, distribution and reproduction in any medium or format, for any purpose, even commercially, as long as you give appropriate credit to the original author(s) and the source, provide a link to the Creative Commons license, and indicate if changes were made.

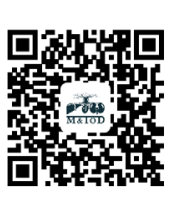




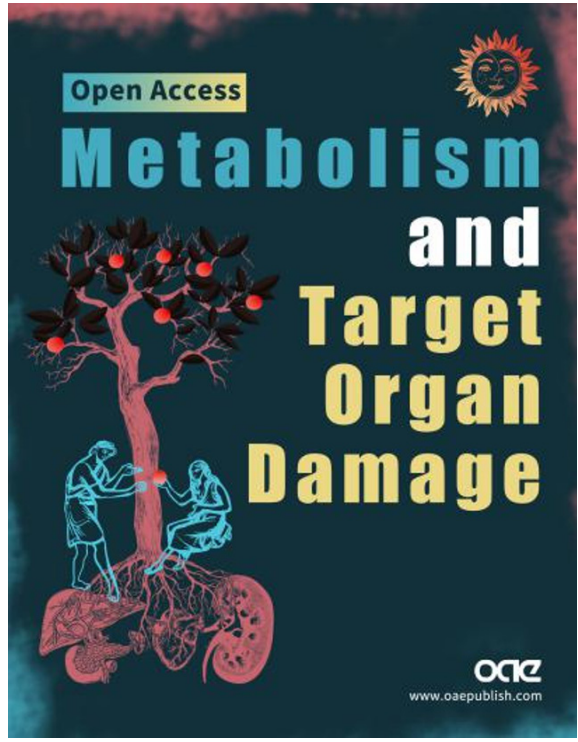

Figure 1. The cover of journal Metabolism and Target Organ Damage.

Aside from the common metabolic syndrome and its individual features, there are more rare inherited metabolic disorders. For example, mitochondrial diseases specifically affect the sub-cellular organelles that produce energy and usually tend to manifest in newborns and children ${ }^{[3]}$.

Therefore, metabolic disorders may occur in all age groups from childhood to adulthood and elderly people are certainly not spared.

Metabolic disorders take a heavy toll on health and well-being of individuals and populations. Data suggest that, in some countries, diabetes and its complications absorb more than $10 \%$ of all health care expenses owing to the direct and indirect costs resulting from hospital inpatient care, overall pharmacy costs, absenteeism, reduced productivity, disability and premature mortality ${ }^{[4]}$.

These figures sound as a wake-up call for addressing metabolic disorders as a major worldwide health issue and a research priority for independent researchers as well as for food and drug industry.

With regard to clinical research, this field is strongly connected with basic and translational science in as much as metabolic pathologies may result from primary endocrine dysfunctions and from the interaction between a genetic predisposition and certain environmental stimuli.

These include, for example, lifestyle changes. Poor eating habits and sedentary behavior are both inherent in western countries and result from population shift. Peoples struggling to adapt from agricultural economies to industrialized areas with their burden of noise and pollution are exposed to increased stress, hypertension, obesity and diabetes ${ }^{[5,6]}$.

Interactions with viral infection (e.g., SARS-Co-V2, HIV and HCV) are also a well defined feature of various metabolic disorders. These may also occur secondary to the use of certain drugs such as those used in psychiatry, rheumatology and oncology.

Of interest, metabolic maladies exhibit sex differences and are also influenced by reproductive status which may be a clue to envisaging personalized metabolic medicine and innovative treatment strategies which are a main focus in our journal's policy. 
Metabolic derangements are a common correlate of ageing, inducing immunological activation and subclinical systemic inflammation, pro-thrombotic phenomena, and increased oxidative stress ${ }^{[7]}$.

A metabolic disorder can develop when some organs, such as liver or pancreas, become anatomically altered or do not function normally. Diabetes is an example. Other examples of cardio-metabolic disorders include target organ damage manifesting as the commonly occurring heart failure, atrial fibrillation, coronary artery disease and ischemic stroke ${ }^{[8]}$.

Besides, chronic kidney disease may result from metabolic derangements such as diabetes and arterial hypertension. It should be highlighted that diabetes is the first cause of blindness and non-traumatic limb amputation in many countries ${ }^{[4]}$.

The development of certain types of cancer, such as liver, breast, pancreas, kidneys, endometrial and colon cancer may be facilitated in the setting of obesity and other metabolic derangements ${ }^{[9]}$.

Increasing evidence demonstrates that metabolic syndrome is also interrelated with many neurodegenerative diseases such as Alzheimer's, Parkinson's and Huntington's Diseases ${ }^{[10]}$.

Relatively more recently, attention has also been paid to the spectrum of fatty liver syndromes that occur in the absence of competing causes of liver disease. Collectively, these were initially defined as NAFLD (i.e., nonalcoholic fatty liver disease) and, more recently, MAFLD (i.e., metabolic-associated fatty liver disease), which is often observed in individuals with diabesity, i.e., diabetes and obesity ${ }^{[11]}$.

Data support the notion that many cases of liver cirrhosis and liver cancer presently occur as a result of metabolic disorders either alone or associated with other noxious stimuli (e.g., heavy alcohol consumption and chronic viral hepatitis).

I was born and educated in southern Italy, working as a clinician in a public hospital in northern Italy and having a long-standing experience in the field of Internal Medicine. I have published more than 200 medical articles in peer-reviewed journals and my $\mathrm{H}$ index is 52 (as indicated by Scopus accessed in March 2021). I am also an expert reviewer (having finished approximately 700 reviews) and I have served or actually serve as a Section, Guest or Associate Editor for other medical journals. As a medical researcher, my personal area of expertise regards the bi-directional association of NAFLD with the metabolic syndrome. This may represent an interesting pathohysiological paradigm for a better understanding of other metabolic disorders.

With this background, I have accepted the privilege to serve as the Editor-in-Chief of Metabolism and Target Organ Damage (M\&TOD).

The idea behind the launch of $M \& T O D$ is to provide an online platform for publication of clinical, basic and translational studies. M\&TOD covers (cardio)-metabolic disorders per se and will specifically emphasize the role of these metabolic conditions as either effectors/amplifiers or consequences of the development and progression of injuries to target organs including but not limited to the cardiovascular system, the liver, kidneys and the pancreas.

We are interested in evaluating submissions pertaining to all areas of public health, pathology, internal medicine and its sub-specialties, general surgery and its branches dealing with epidemiology, physiopathology, prevention, diagnosis, and prognosis and management of systemic and organ metabolic disorders. The journal welcomes submissions of all the editorial categories from case studies to original 
articles, reviews, editorials, commentaries and meta-analyses, irrespective of the patients' age groups, from pediatrics to geriatrics. Priority will be given on the grounds of originality and translational impact of submitted articles. A specific feature of our editorial policy is to promote animal-free studies which use, for example, cell cultures and artificial intelligence. In a subordinate position to animal-free studies, we endorse research which tries to maximize wellbeing will avoid pain and distress in animals. Additionally, we strongly support equal gender representation in publishing. Young investigators and researchers from all over the world especially from developing countries are welcome.

I am grateful to those distinguished scholars who have accepted to participate in the Editorial Board: their personal scientific prestige is a strong determinant of our journal credibility.

The Editorial Board of this journal is committed to working hard to guarantee accurate and fair evaluation and prompt publication of papers received, with the aim of creating a scientifically solid and successful scientific journal.

Thank you.

Amedeo Lonardo, M.D.

Metabolism and Target Organ Damage Editor-in-Chief

\section{DECLARATIONS}

\section{Authors' contributions}

Preparing the manuscript draft: Lonardo A

\section{Availability of data and material}

Not applicable.

\section{Financial support and sponsorship}

None.

\section{Conflicts of interest}

The author declared that there are no conflicts of interest.

\section{Ethical approval and consent to participate}

Not applicable.

\section{Consent for publication}

Not applicable.

\section{Copyright}

(c) The Author(s) 2021.

\section{REFERENCE}

1. Mittendorfer B. Origins of metabolic complications in obesity: adipose tissue and free fatty acid trafficking. Curr Opin Clin Nutr Metab Care 2011;14:535-41.

2. Romeo GR, Lee J, Shoelson SE. Metabolic syndrome, insulin resistance, and roles of inflammation--mechanisms and therapeutic targets. Arterioscler Thromb Vasc Biol 2012;32:1771-6.

3. Gorman GS, Chinnery PF, DiMauro S, et al. Mitochondrial diseases. Nat Rev Dis Primers 2016;2:16080.

4. The Congressional Diabetes Caucus Release. About Diabetes. Available from: https://diabetescaucus-degette.house.gov/facts-and-figures. [Last accessed on 12 Mar 2021] 
5. Ramachandran A, Mary S, Yamuna A, Murugesan N, Snehalatha C. High prevalence of diabetes and cardiovascular risk factors associated with urbanization in India. Diabetes Care 2008;31:893-8.

6. Münzel T, Sørensen M, Schmidt F, et al. The adverse effects of environmental noise exposure on oxidative stress and cardiovascular risk. Antioxid Redox Signal 2018;28:873-908.

7. Ferrucci L, Fabbri E. Inflammageing: chronic inflammation in ageing, cardiovascular disease, and frailty. Nat Rev Cardiol 2018;15:50522.

8. Gerdts E, Regitz-Zagrosek V. Sex differences in cardiometabolic disorders. Nat Med 2019;25:1657-66.

9. Calle EE, Kaaks R. Overweight, obesity and cancer: epidemiological evidence and proposed mechanisms. Nat Rev Cancer 2004;4:57991.

10. Yalcin G, Yalcin A. Metabolic syndrome and neurodegenerative diseases. J Geriatr Med Gerontol 2018;4:042.

11. Lonardo A. Renaming NAFLD to MAFLD: Could the LDE system assist in this transition? J Clin Med 2021;10:492. 